Ibn Al-Haitham Jour. for Pure \& Appl. Sci. 35(1)2022

Ibn Al Haitham Journal for Pure and Applied Science

Journal homepage: http://jih.uobaghdad.edu.iq/index.php/j/index

\title{
Design of Truncated Hyperboloid Solar Concentrator by Using Zemax Program
}

\author{
Hassanein Nouri Hamza \\ Hasanein.Nouri1204a@ihcoedu.uobaghdad.edu.iq
}

\author{
Alaa Badr Hasan \\ alaajizany@yahoo.com
}

Department of physics, College of Education for Pure Sciences, University of Baghdad, Iraq.

Article history: Received 9, May, 2021, Accepted 13, July, 2021, Published in January 2022.

Doi: $10.30526 / 35.1 .2780$

\begin{abstract}
A hyperboloid solar concentrator (HSC) was designed with a truncation angle side to the entrance aperture by simulation in the Zemax Optical Design software. The (HSC) has a wide exposure range to solar radiation due to the relatively large entrance aperture. The design consists of an entrance aperture facing the sun to obtain the largest possible amount of solar radiation, and a small exit aperture compared to the entrance aperture whose mission is to receive solar radiation after it enters the entrance aperture and reflects it from the inner reflective walls of the (HSC). In the exit aperture, there is a detector to measure the number of rays incident on it, which is a measure of the optical efficiency of the sample. A hybrid sample has been designed with an angular truncation at the front of it. This feature contributes to a greater absorption of solar radiation for greater efficiency. The results showed a slight change in the value of optical efficiency when changing the truncation angle, and the values of optical efficiency were superior to designs that had a small concentration ratio, and optical efficiency increased with increasing the concentrator length.
\end{abstract}

Keywords: Solar Concentrator, truncated hyperboloid, Acceptance Angle, Concentration ratio, Zemax

\section{Introduction}

The topic of solar concentrators has great importance because of consideration of the exploitation of solar energy. The solar concentrators intercept solar radiation and convert it into another form of thermal or electrical energy. The need to use solar concentrators as an 


\section{Ibn Al-Haitham Jour. for Pure \& Appl. Sci. 35(1)2022}

important part of renewable energy production has increased, and thus it has a prominent role in compensating the traditional energy and preserving the environment from pollution resulting from fossil fuels [1].

The desirable characteristics of solar concentrators are prolonged use without the need for a solar tracking system. Due to the industrial and engineering complexities of these systems, the solar center has the ability to reflect the solar radiation to the receiver with different angles of incidence until it reaches the acceptance angle that gives an acceptable optical efficiency $(80 \%)$ [2].

The solar concentrators consist of major parts: it is the reflecting surface that reflects and redirects solar rays to the receiver that collects and stores solar energy, the entrance aperture that controls the amount of radiation entering the concentrator, and the solar tracking system that contributes to directing the concentrator to the direction of the sun to obtain the largest amount possible from solar radiation [3].

The concentration ratio, defined as the ratio between the areas of the entrance aperture to the area of the exit aperture, has a major role in the performance of the solar concentrator. This percentage increases to more than one in most solar concentrators, especially in large projects that produce high electrical energy [4].

The concentration ratio has the range $(1-2)$ in the most solar collectors. Where increasing in the concentration ratio is associated with the increase in the temperature acquired by the sun. While the concentration that have a higher concentration of solar ovens are used in studying materials at high temperatures.

Solartrough concentrators are commonly used in the field of solar energy exploitation due to the peculiarity of this design in terms of ease of manufacture, transportation and high efficiency. These concentrators are distinguished by their different shapes according to the purpose of the design, as they are in the form of a rectangular trough or a collector with a conical cross (parabolic or hyperbolic) or dish [5].

The hyperbolic concentrator is one of the important designs that have taken a great deal of attention and research by designers and researchers.I. M. Salehet. focused on the optical performance evaluation of a 3-D Elliptical Hyperboloid Concentrator (EHC) designed by using ray tracing tool, the OpticsWorks software. An extensive theoretical ray tracing technique has been adopted to calculate the optical efficiency of the novel 3-D EHC. The performance parameters such as optic concentration factor, optical efficiency and geometric concentration ratio are also evaluated for different aspect ratios of the elliptical profile [6].

V.Chittalakkotteet. al. attempt is made to design, develop and experimentally analyze the performance of adesalination unit with a hyperboloid concentrator and a helical copper coil receiver. The elliptic hyperboloidconcentrator is a special design for concentrating and capturing radiation over a wide incidentangle and thus eliminates tracking. Further a CFD analysis was carried out to design and optimize thehelical coil receiver dimensions. The optimized receiver was then fabricated [7].

K.S. Reddyet. al. use an elliptic hyperboloid concentrator and a helical receiver along with a multi-tray desalination unit to purify water in themost effective manner. The helical receiver proposed in the present work aims at the Dean Flow effect in order to enhance heat transfer inlaminar flow. The effectiveness of this property with respect to various physical parameters has been observed and an optimum design has been suggested based on this [8]. 


\section{Optical design}

The solar center consists of an entrance aperture, capturing the solar radiation and redirecting it into the solar concentrator system. An exit aperture of thermal warehouse is placed to transfer heat to a suitable place to be used for the purposes of designing the concentrator [9]. The concentrator also consists of reflective inner walls with a hyperbola shape to redirect the solar radiation to the receiver. This sample is characterized by the presence of an angular truncation of the front edge of concentrator. The function of this truncation is to extend the range of ray capture by the entrance aperture. Figure (1) illustrates the scheme of hyperbolic solar concentrator. The design has two focuses (B, B') that collect the reflected radiation from inner surfaces of the (HSC). Figure (2) illustrates 3D layout of truncated (HSC) that is provided by the Zemax program to give the designer flexibility in using the tools, giving a broad idea about the design and handling errors.

The concentration ratio of the design has been changed to the values of $(C=1,2,3 \ldots 10)$, which are considered one of the important factors affecting the design performanceby controlling the amount of rays entering and leaving it. As for the angle of truncation, specific values were chosen within the range $\left(\theta=10^{\circ}, 20^{\circ}, 30^{\circ}, 40^{\circ}, 50^{\circ}\right)$ to show the effect of this efficiency angle of the optical system. The length of concentrator has been changed the range $(100,200,300,400,500 \mathrm{~mm})$ to evaluate the system performance. The length of the concentrator controls the amount of internal reflections of the rays at the inner surfaces, as well as the concentration ratio because the geometrical design of the (HSC) limits this ratio according to the length of the (HSC).

The angle of incidence is the main criterion for determining the performance of solar concentrators, because it is the main factor discovering the amount of radiation entering the system. Changing is according to the angle of incidence throughout the period of daylight hours due to the change in the position of the sun in the sky. The importance of the acceptance angle is that it is used in solar concentrators that do not contain a solar tracking system. The important factor is the time period in using the solar concentrator at daytime and its rush hour, which gives the maximum efficiency.

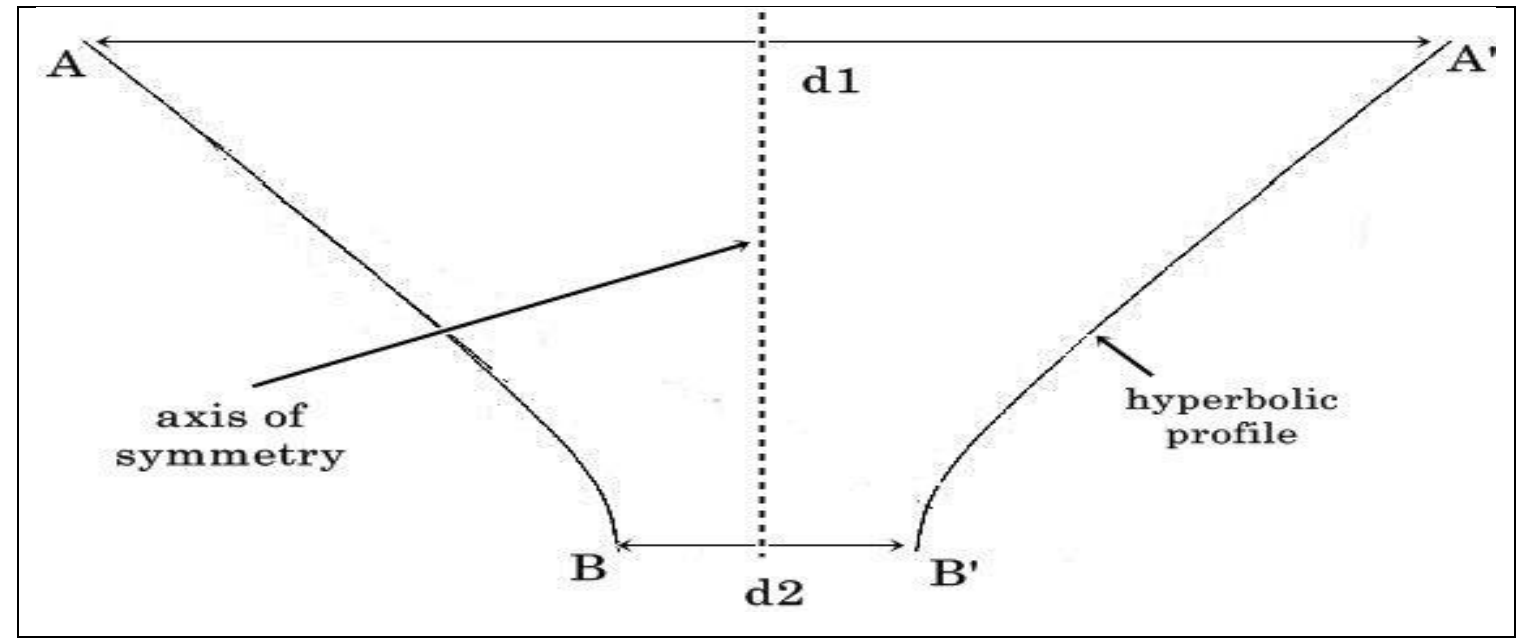

Figure1. Scheme of hyperbolic solar concentrator [10] 
Ibn Al-Haitham Jour. for Pure \& Appl. Sci. 35(1)2022

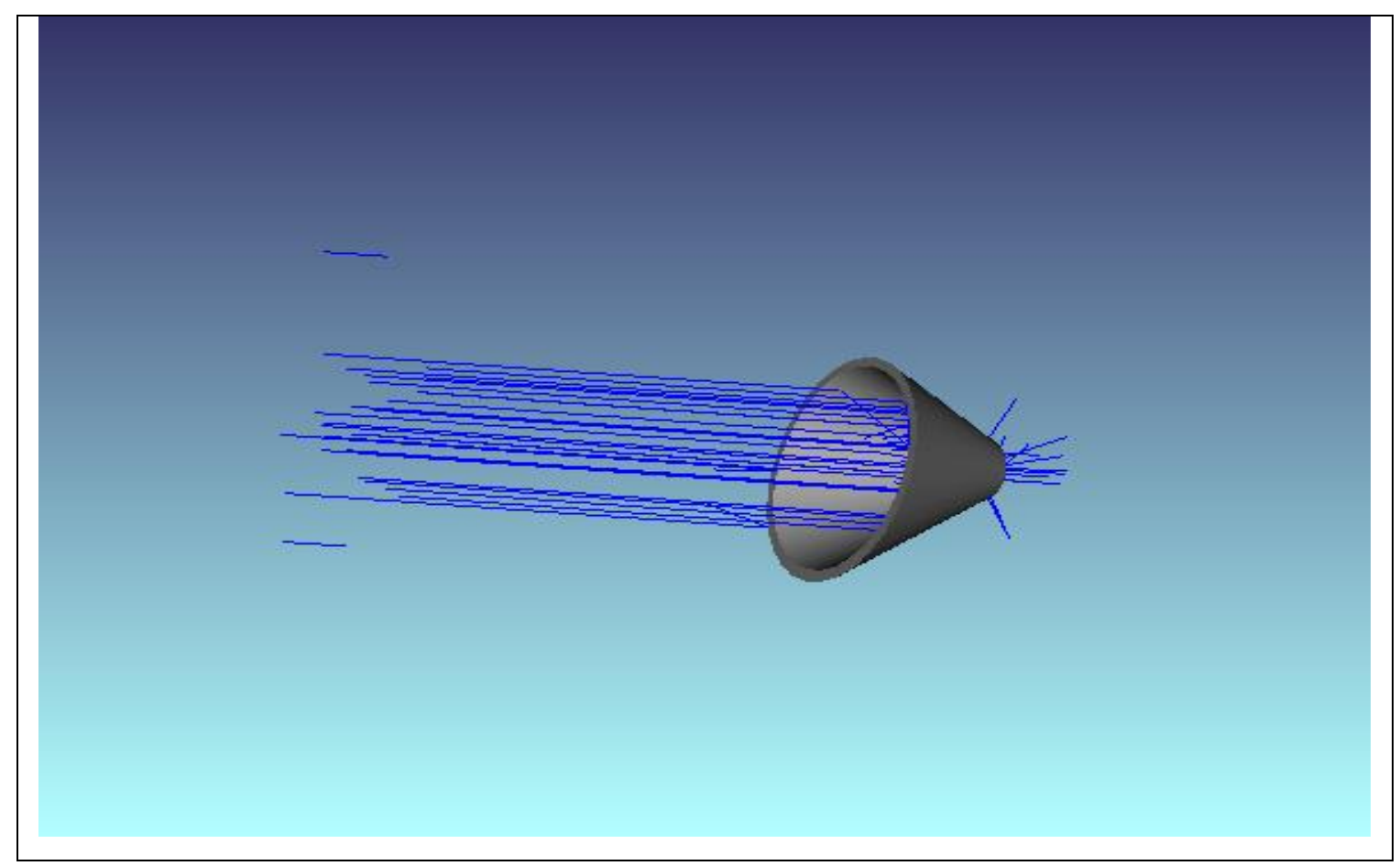

Figure2. 3D layout of truncated hyperbolic solar concentrator in Zemax program

\section{Results}

Figure (3) shows the optical efficiency of the design when the angle of incidence changes to different values of the truncation angle of the design. The figure shows a slight change in the efficiency curve when the truncationangle changes, although we note the preference for small truncationangles. The reason for this function to behave is due to the decrease in the internal surface area of the reflector when the truncationangle is increased. So the amount of wasted radiation will be increased. In general, the figure shows the efficiency slope clearly when the angle of incidence changes, as the design gives a value of the acceptance angle equal to $\left(3^{\circ}\right)$ approximately for all the samples used.

Figure (4) shows the optical efficiency of the design when the angle of incidence changes into different values of the concentration ratio. The figure illustrates the preference of the low concentration ratios $(C=1-5)$ over the rest of the values $(C=6-10)$. The efficiency begins in the first samples with high efficiency $(100 \%)$, while the other samples, the efficiency decreases to less than $(50 \%)$. The reason behind this behaviour is that the geometric design of the sample, which has a low concentration ratio, has a little inclination in its reflecting walls, which leads to the reflection of the incoming rays at a large angle that allows it to be delivered to the exit aperture without many repeated reflections. This reduces the lost radiation. Consequently, it increases the amount of radiation received by the receiver, thus it leads to increase the efficiency.

Figure (5) shows the optical efficiency when the angle of incidence changes into different values of concentration length. The figure shows the extent to which the optical efficiency is affected by the concentration length, where the sample that has a greater length gives greater efficiency. Increasing the concentration length gives a greater opportunity to capture the largest possible number of solar rays to reach the receiver. This illustrates the behaviour of the function in Figure (5). However, the impact of the angle of incidence remains obvious on the optical efficiency because it is the most important factor in determining the efficiency of all solar concentrators. 
Ibn Al-Haitham Jour. for Pure \& Appl. Sci. 35(1)2022

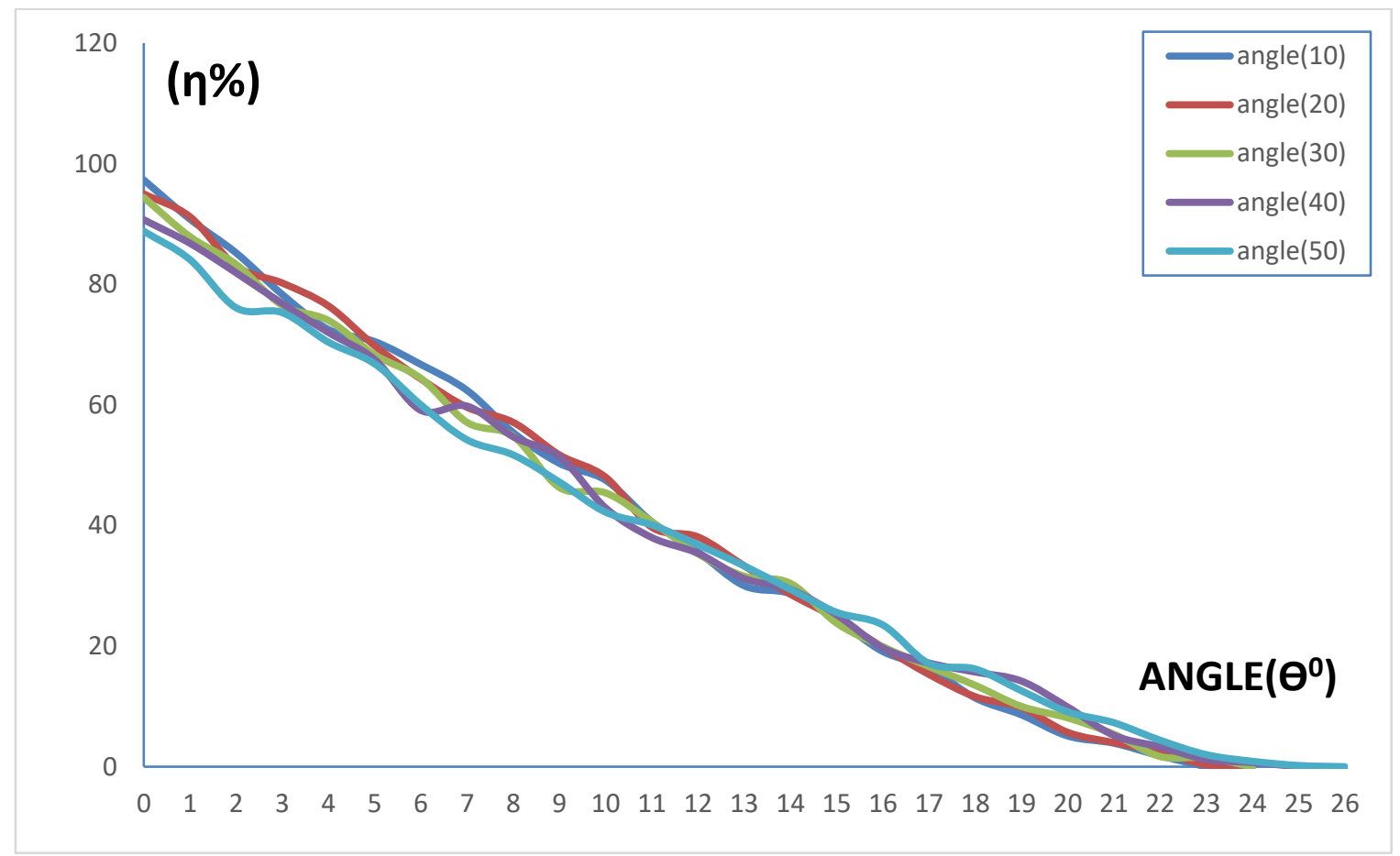

Figure3. The optical efficiency of (HSC) at different incident angle for samples have different truncation angles

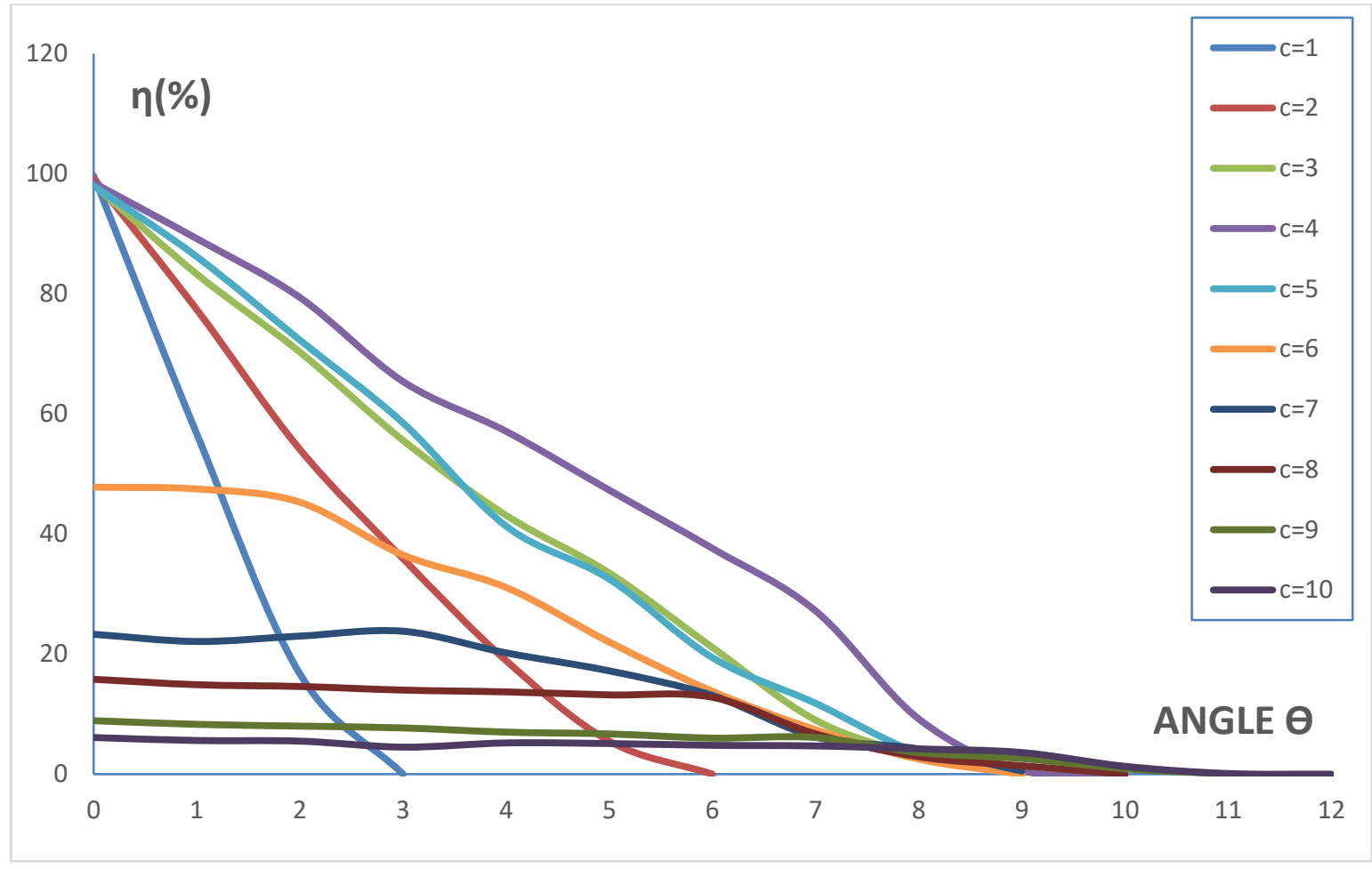

Figure 4. The optical efficiency of (HSC) at different incident angle for samples have different concentration ratio 
Ibn Al-Haitham Jour. for Pure \& Appl. Sci. 35(1)2022

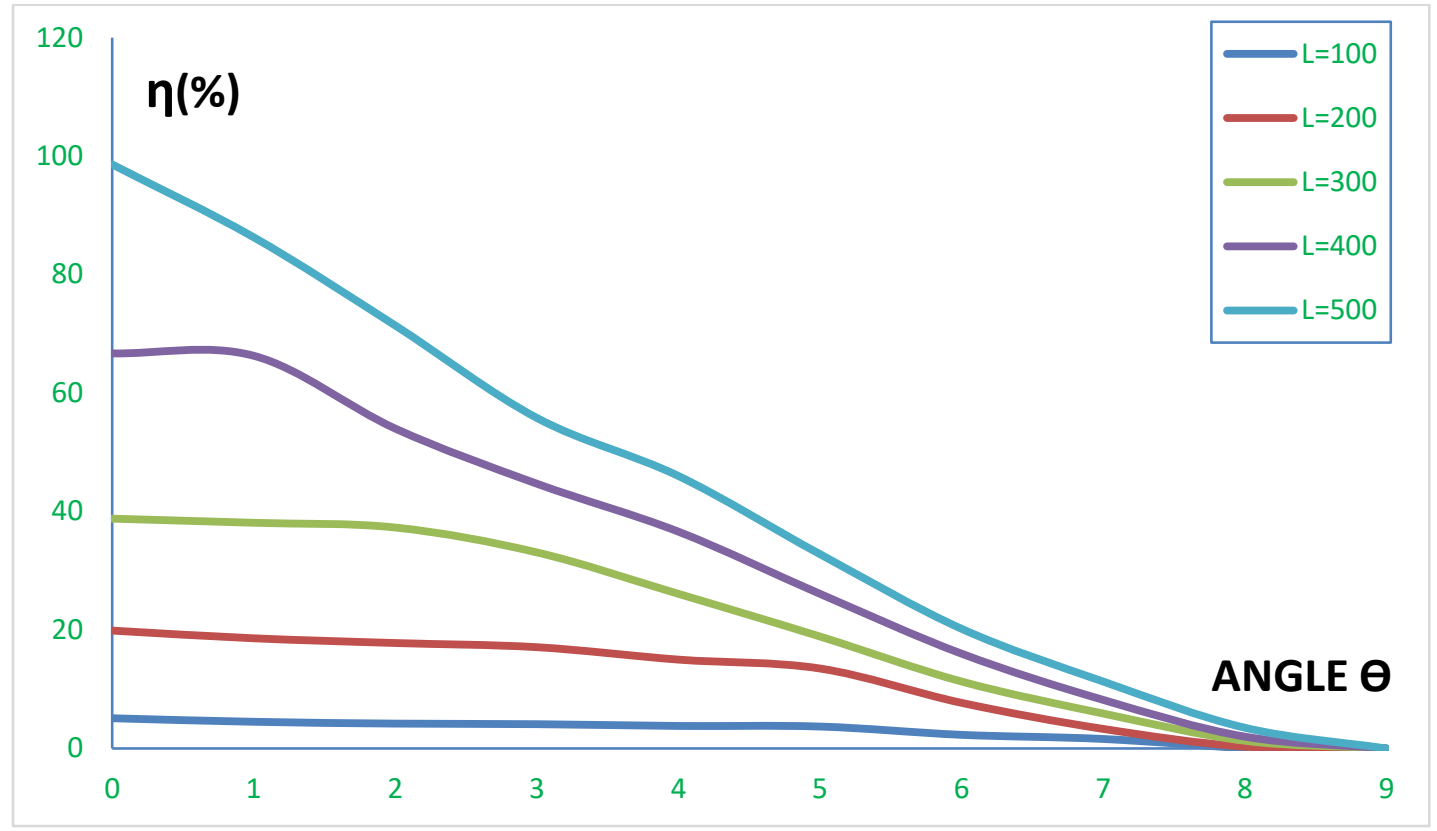

Figure 5. The optical efficiency of (HSC) at different incident angle for samples has different concentration length

\section{Conclusion}

The design shows priority of first sample comparing with other samples. This feature contributes to a greater absorption of solar radiation for greater efficiency. The results showed a slight change in the value of optical efficiency when changing the truncation angle, the values of optical efficiency were superior to designs that have a small concentration ratio, and optical efficiency increased with increasing concentrator length.

\section{References}

1. Islam, M. T.; Huda N.; Abdullah, A. B.; Saidur R. A comprehensive review of stateof-the-art concentrating solar power (CSP), Renew. Sustain. Energy Rev. 2017, 91. 987-1018.

2. O'Gallagher, J. J. Nonimaging Optics in Solar Energy. Synth. Lect. Energy Environ. Technol. Sci. Soc. 2008, 2, 1-12.

3. Atheaya, D.; Tiwari, G. N. Review on solar thermal power concentrators, MOJ Sol. Photoenergy Syst. 2017, 3, 23-33.

4. Garcia, A.; Fernandez, A. A.; Vázquez D.; Bernabeu E. Ideal 3D asymmetric concentrator. Sol. Energy, 2009, 83,1, 113-117.

5. Saleh, I. M.; O’Donovan, T. S.; Reddy, K. S.; Mallick, T. K. An optical analysis of a static 3-D solar concentrator. Sol. Energy, 2013, 88, 57-70.

6. Saleh, I. M.; Vikram, T. S.; O’Donovan, T. S.; Reddy, K. S.; Mallick, T. K. Design and experimental analysis of a static 3-D elliptical hyperboloid concentrator for process heat applications. Sol. Energy. 2014, 102, 257-266.

7. Chittalakkotte, V.; Vincent, V. L.; Valiyaparambil, P. Development of a solar energy based desalination system using a hyperboloid concentrator. Mater. Today Proc. 2020,22,1-15. 
8. Imhamed, M. S.; Mallick, T. K.; Kew, P. A.; O’Donovan, T. S.; Reddy, K. S. Optical performance evaluation of a 2-D and 3-D novel hyperboloid solar concentrator. World Renewable Energy Congress XI, Abu Dhabi, UAE, 2010, 21, 1-16,

9. Ameer, S. A.; Shahad, H. A. Characteristics Review of Optical Concentrators. Int. J. Curr. Eng. Technol. 2017, 7, 1, 168-182,

10. Khamooshi, M.; Salati, H.; Egelioglu, F.; Faghiri, A. H.; Tarabishi, J.; Babadi, S. Review of Solar Photovoltaic Concentrators. Hindawi Publishing Corporation International Journal of Photo energy. 2014, 11, 17-22. 